\title{
Optimum phase noise reduction and repetition rate tuning in quantum-dot mode- locked lasers
}

Cite as: Appl. Phys. Lett. 104, 021112 (2014); https://doi.org/10.1063/1.4861604

Submitted: 01 December 2013 • Accepted: 21 December 2013 • Published Online: 15 January 2014

T. Habruseva, D. Arsenijević, M. Kleinert, et al.
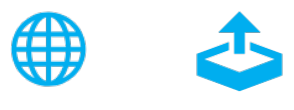

\section{ARTICLES YOU MAY BE INTERESTED IN}

Phase noise and jitter reduction by optical feedback on passively mode-locked quantum-dot lasers

Applied Physics Letters 103, 231101 (2013); https://doi.org/10.1063/1.4837716

Hybrid mode-locking in a $40 \mathrm{GHz}$ monolithic quantum dot laser

Applied Physics Letters 96, 011104 (2010); https://doi.org/10.1063/1.3279136

Perspective: The future of quantum dot photonic integrated circuits

APL Photonics 3, 030901 (2018); https://doi.org/10.1063/1.5021345

田QBLOX

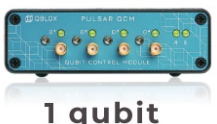

Shorten Setup Time

Auto-Calibration

More Qubits

Fully-integrated

Quantum Control Stacks

Ultrastable DC to $18.5 \mathrm{GHz}$

Synchronized $<<1$ ns

Ultralow noise

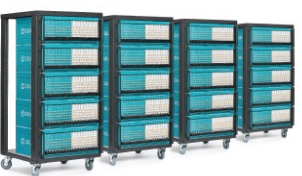

100s qubits

visit our website > 


\title{
Optimum phase noise reduction and repetition rate tuning in quantum-dot mode-locked lasers
}

\author{
T. Habruseva, ${ }^{1,2,3}$ D. Arsenijević, ${ }^{4}$ M. Kleinert, ${ }^{4}$ D. Bimberg, ${ }^{4}$ G. Huyet,,${ }^{1,2}$ \\ and S. P. Hegarty ${ }^{1,2}$ \\ ${ }^{1}$ CAPPA, Cork Institute of Technology, Cork, Ireland \\ ${ }^{2}$ Tyndall National Institute, Lee Maltings, Cork, Ireland \\ ${ }^{3}$ Aston University, Aston Triangle, B4 7ET Birmingham, United Kingdom \\ ${ }^{4}$ Institut für Festkörperphysik, Technische Universität Berlin, Berlin, Germany
}

(Received 1 December 2013; accepted 21 December 2013; published online 15 January 2014)

\begin{abstract}
Competing approaches exist, which allow control of phase noise and frequency tuning in modelocked lasers, but no judgement of pros and cons based on a comparative analysis was presented yet. Here, we compare results of hybrid mode-locking, hybrid mode-locking with optical injection seeding, and sideband optical injection seeding performed on the same quantum dot laser under identical bias conditions. We achieved the lowest integrated jitter of $121 \mathrm{fs}$ and a record large radio-frequency (RF) tuning range of $342 \mathrm{MHz}$ with sideband injection seeding of the passively mode-locked laser. The combination of hybrid mode-locking together with optical injectionlocking resulted in $240 \mathrm{fs}$ integrated jitter and a RF tuning range of $167 \mathrm{MHz}$. Using conventional hybrid mode-locking, the integrated jitter and the RF tuning range were $620 \mathrm{fs}$ and $10 \mathrm{MHz}$, respectively. (C) 2014 AIP Publishing LLC. [http://dx.doi.org/10.1063/1.4861604]
\end{abstract}

Monolithic semiconductor mode-locked lasers (MLLs) emit series of narrow pulses of light at high repetition rates ranging from 5 to $80 \mathrm{GHz} .{ }^{1,2}$ Optical frequency combs emitted by MLLs can be utilized in a variety of applications. Arbitrary waveform generation, orthogonal frequencydivision multiplexing, and coherent wavelength division multiplexing techniques need stable frequency combs of narrow optical linewidth, while short periodic pulses with low level of pulse-to-pulse timing fluctuations are required in optical time-division multiplexing, optical sampling, and alloptical clock recovery.

MLLs based on InAs/GaAs quantum-dots (QDs) have demonstrated advantageous performance characteristics when compared to quantum well lasers, such as generation of ultrashort pulses, ${ }^{3}$ thermal stability, ${ }^{4,5}$ and low jitter below $10 \mathrm{fs}$ per cycle, ${ }^{6,7}$ which make them suitable candidates for use as optical sources in optical communication systems. ${ }^{8}$ However, outside the optimal parameter range, frequency combs generated by passively MLLs (PMLLs) exhibit large phase noise and do not meet jitter requirements, which makes them ineffective in high-speed applications and optical sampling. Therefore, utilization of QD-PMLLs in these applications requires the introduction of noise stabilization techniques.

An important consideration for the production of deployable sources is the device cleaving error. A typical variation of $5 \mu \mathrm{m}$ in the device length results in a $200 \mathrm{MHz}$ pulse repetition rate shift for a $40 \mathrm{GHz}$ laser which poses a difficulty for clocked systems. The mode-locking frequency can also vary significantly with bias conditions and temperature. ${ }^{9}$ Therefore, universal techniques allowing the tuning of the device repetition rate become essential to compensate these variations and match device repetition rate to the predefined frequency of the system.

This Letter provides an overview and experimental comparison of several techniques, allowing noise reduction and tuning of the device fundamental frequency, $F_{r e p}$, in MLLs.
For the relevant comparison of stabilization techniques, all experimental investigations were performed on the same device at a fixed temperature and similar bias conditions. We used a monolithic two-section QD laser of $1 \mathrm{~mm}$ length $\left(F_{\text {rep }}=39.5 \mathrm{GHz}\right)$. The active zone of the device consisted of 15 layers of InAs QDs emitting at $1.3 \mu \mathrm{m}$ embedded in InGaAs quantum wells. The saturable absorber of $100 \mu \mathrm{m}$ length was separated from the gain section by an etched $20 \mu \mathrm{m}$ gap, giving an electrical isolation of $\sim 10 \mathrm{k} \Omega$ between the sections. The facet next to the absorber was coated for high reflectivity of $95 \%$, the facet next to the gain section was as cleaved $(\mathrm{R} \sim 32 \%)$. The laser was integrated into a module comprising a thermo-electric cooler, a single-mode fiber pigtail, and an impedance matched microwave port. The temperature was set at $21^{\circ} \mathrm{C}$, the device absorber section was reverse biased at $-8.0 \mathrm{~V}$, and the gain current was around $60 \mathrm{~mA}$ in all measurements. The laser output was characterized by optical and electrical spectrum analyzers (OSA and ESA, respectively) and an autocorrelator (AC).

The PMLL emitted pulses of 2.8 ps duration with optical spectrum width of $10 \mathrm{~nm}$ at $-10 \mathrm{~dB}$ level. Figure 1 shows an optical spectrum (a), an autocorrelation trace (b), and a radio-frequency (RF) spectrum (c) of the laser when passively mode-locked. The autocorrelation traces were fitted well to a Gaussian (Figure 1(a), red).

The QD-MLL demonstrated significant phase noise with optical modal linewidth values in the range of $100 \mathrm{MHz}$ and large integrated jitter of $\sim 9$ ps (retrieved from single- sideband noise and integrated from $10 \mathrm{kHz}$ to $1 \mathrm{GHz}$ ) when operating in a passive mode-locked regime.

The reduction of jitter and RF frequency tuning can be achieved by the electrical modulation of the absorber reverse bias, "hybrid" mode-locking (HML). Since the first demonstration of hybrid mode-locking in a $10 \mathrm{GHz}$ QD-MLL, (Ref. 10) a significant reduction of the pulse jitter ${ }^{11}$ and a repetition rate control within a $30 \mathrm{MHz}$ tuning range ${ }^{12}$ have been achieved. 

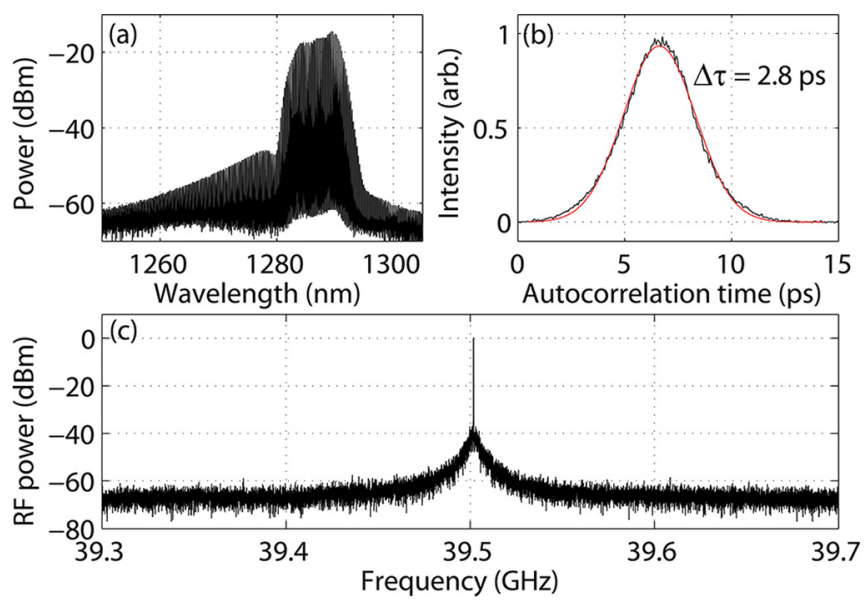

FIG. 1. (a) Optical spectrum, (b) autocorrelation trace (black) with Gaussian fit (red), (c) RF spectrum of the PMLL.

For hybrid mode-locking, the voltage applied to the device absorber section combined a DC component $\mathrm{V}$ and a $40 \mathrm{GHz}$ low noise signal in the form of $\mathrm{V}+\mathrm{ACos}(\Omega \mathrm{t})$, where A and $\Omega$ are the amplitude and frequency of modulation of the reverse bias, respectively.

Hybrid mode-locking allowed tuning of the device repetition rate by changing $\Omega$. The locking boundaries measured versus the modulation power for the chosen bias conditions are shown in Figure 2(a); the laser repetition rate followed the modulation frequency in the range shown in grey. We considered the laser as locked to the external modulation when the fundamental frequency of mode-locking followed the modulation frequency $\Omega$ with sideband suppression ratio of $>30 \mathrm{~dB}$. Similarly to the previously published results, ${ }^{12}$ the laser exhibited an asymmetrical locking range, wider at frequencies lower than the passive mode-locking frequency. The maximum tuning range of $\sim 10 \mathrm{MHz}$ was achieved at maximum applied modulation power of $14 \mathrm{dBm}\left(3.2 \mathrm{~V}_{\mathrm{pp}}\right)$. Synchronization to the external clock resulted in the RF linewidth narrowing to the values below resolution bandwidth of the ESA $(1 \mathrm{kHz})$. The device integrated timing jitter decreased with $\mathrm{V}_{\mathrm{pp}}$ down to a minimum value of $620 \mathrm{fs}$ (Figure 2(b)).

Hybrid mode-locking did not significantly affect the optical spectrum and autocorrelation traces; they were close to those of the passive mode-locked regime and practically unchanged for all studied modulation amplitudes and
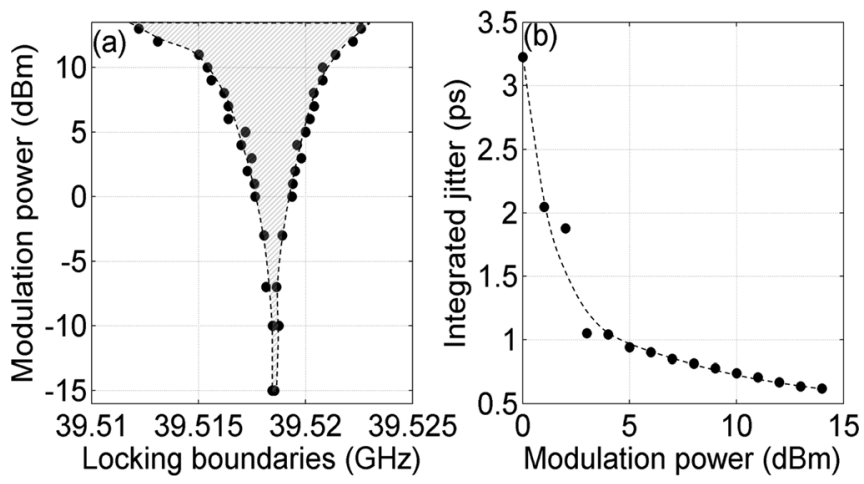

FIG. 2. (a) Measured boundaries of the $F_{\text {rep }}$ tuning range versus modulation power. (b) Integrated timing jitter versus modulation power. frequencies with spectral widths of $10 \mathrm{~nm}$ at $-10 \mathrm{~dB}$ level, and pulse width of $3.1 \mathrm{ps}$. The modal optical linewidths were also similar to the PMLL.

External optical injection has been shown to be an effective method for noise reduction in semiconductor MLLs. The combination of single-tone $\mathrm{CW}$ injection and active/hybrid mode-locking resulted in optical linewidth reduction below $20 \mathrm{kHz}$ (Ref. 13) and phase locking of all slave modes to the master laser in QW devices. ${ }^{14,15}$ Excited state pulse injection into a QD-PMLL oscillating in the ground state band demonstrated phase and amplitude noise reduction of the slave laser. ${ }^{16}$ Stable injection-locking with no instabilities observed at zero detuning frequency has been demonstrated in QD single section and PMLLs. ${ }^{17-19}$ These observations suggest that the noise characteristics of QD hybrid mode-locked lasers (HMLLs) could be further improved by optical injection seeding.

For $\mathrm{CW}$ injection, the light from a tunable laser (master laser) was injected through an optical fiber circulator into the gain section of the QD-HMLL (slave laser). A polarization controller was used to match the polarization of the injection seed and the slave. The master laser had a narrow optical linewidth of $\sim 200 \mathrm{kHz}$.

As shown in Figure 3(a), when the slave laser was locked to the master its optical spectrum was narrowed with the majority of the power red shifted from the injection wavelength, here $1288.44 \mathrm{~nm}$. Optical spectrum narrowing led to pulse broadening: the autocorrelation traces in Figure 3(b) show Gaussian shape with deconvolved pulse width of 4.8 ps. The pulse train had a higher DC level than for the PMLL due to large injection power, but still well distinguished pulses.

We measured the common-mode optical linewidth of the slave using a self-heterodyne technique: the slave output was split in a 50/50 fiber coupler, one part of the signal was shifted by $80 \mathrm{MHz}$ via an acousto-optic modulator and then recombined with the other part. A $10 \mathrm{~km}$ fiber spool was included to break the coherence between the laser outputs. We added a variable delay to adjust the pulse arrival time and a polarization controller to match polarization in both
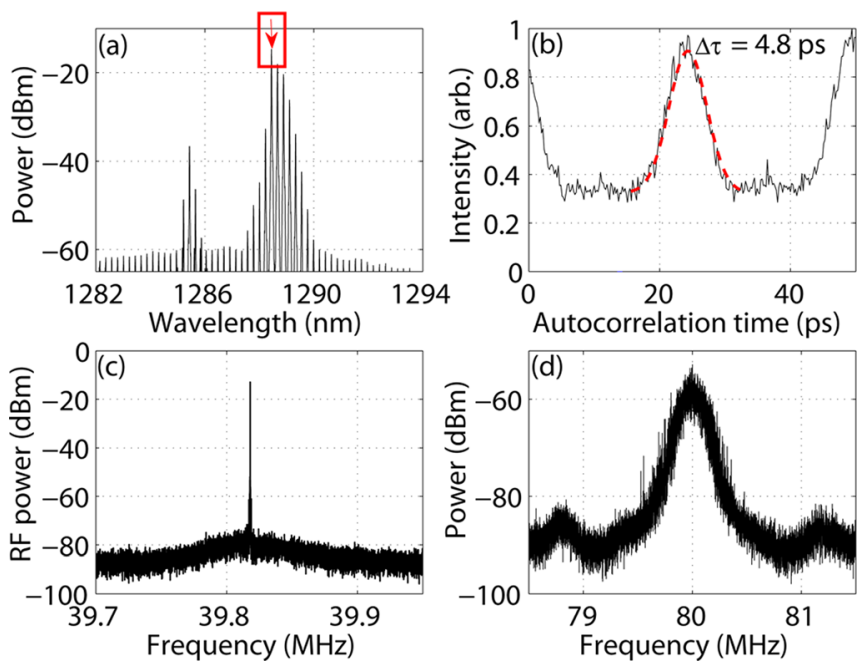

FIG. 3. (a) Optical spectrum of the injection-locked HMLL. Injection seed is shown with an arrow. (b) Autocorrelation trace. (c) RF spectrum. (d) Selfheterodyne beating signal. Injection power $\mathrm{P}_{\mathrm{inj}}=770 \mu \mathrm{W}$; injection wavelength of $1288.44 \mathrm{~nm}$, absorber bias modulation power of $14 \mathrm{dBm}$. 
branches. For $14 \mathrm{dBm}$ modulation amplitude, and injection power and wavelength of $770 \mu \mathrm{W}$ and $1288.44 \mathrm{~nm}$, the beating signal linewidth found from the Lorentzian fit was $\sim 200 \mathrm{kHz}$, demonstrating complete optical linewidth reduction for the slave (Figure 3(d)). With injection seed, the integrated jitter was reduced by a factor of $\sim 3$, down to 240 fs. The example of the RF spectrum within the locking range boundaries is shown in Figure 3(c).

Due to the reduction of the slave spectral width, injection locking resulted in the increase of the $F_{\text {rep }}$ tuning range when compared to the free-running HMLL, which is consistent with previously published theoretical results. ${ }^{12}$

Figure 4 shows RF spectra of the injected HMLL for a range of modulation frequencies, demonstrating the largest $F_{\text {rep }}$ tuning range of $167 \mathrm{MHz}$. In the case of reduced modulation amplitude of $9 \mathrm{dBm}$ while the injection settings are unchanged, the locking range decreased down to $40 \mathrm{MHz}$ and the integrated jitter increased to a value of $365 \mathrm{fs}$. This shows that both the optical injection and HML have an influence on the laser emission (unlike the sidebands injectionlocking of the PMLL discussed in the next paragraph, where the injection dominates within the locking range).

Injection-locking via two coherent lines, generated by modulation of a CW master source, ${ }^{20}$ has been shown to be a successful technique to improve noise properties in $10 \mathrm{GHz}$ QD-MLLs, resulting in improved control of the pulse repetition rate, wavelength tuning, all-modal linewidth reduction, and decrease of timing jitter, along with pulse stabilization and chirp reduction. ${ }^{21,22}$ In this section, we discuss the outcomes of the sideband injection locking for the QD-PMLL under test and compare them with stabilization techniques discussed above.

For dual-tone injection, the light from a tunable laser was modulated by a Mach-Zehnder amplitude modulator biased at the transmission minimum and driven via a lownoise signal generator at $F_{\text {rep }} / 2$, giving two coherent sidebands separated by $F_{\text {rep }}$ with a suppressed carrier frequency. As before, the master emission was coupled to the gain section of the laser through a fibre-optic circulator.

Similar to the CW injection seeding, dual-tone injection resulted in the optical spectrum narrowing and red shifting and the pulse width broadening. Self-heterodyne measurements demonstrated narrow beating signal with $\sim 135 \mathrm{kHz}$ full width at half maximum, thus showing all-modal optical

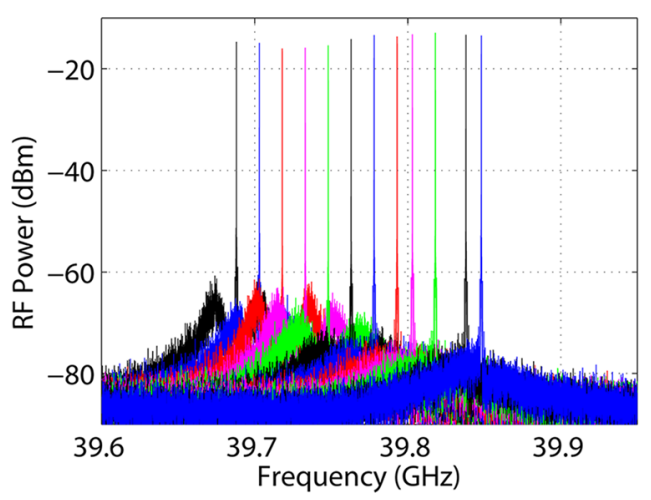

FIG. 4. RF spectra of the injection-locked QD-HMLL for a range of modulation frequencies.
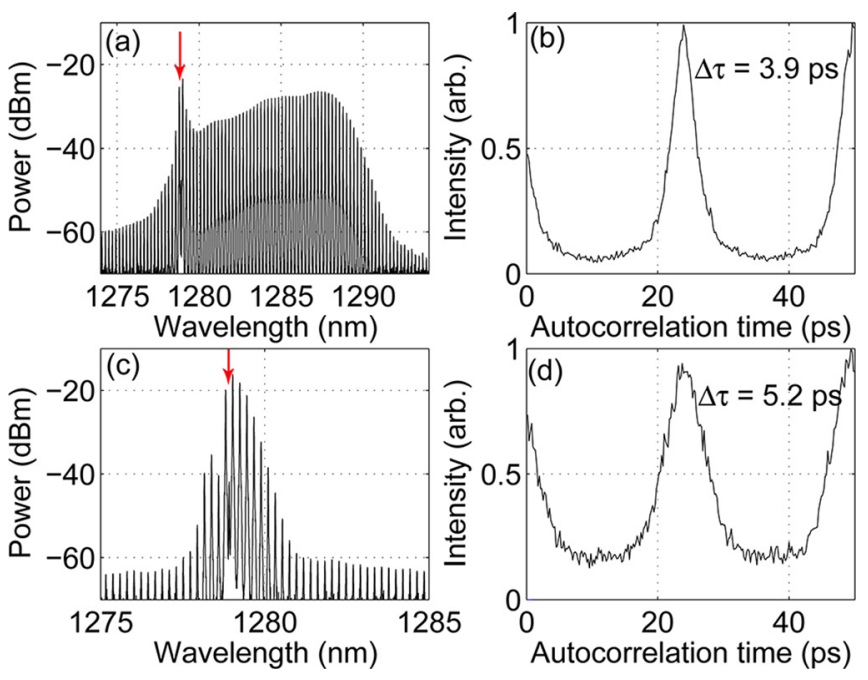

FIG. 5. (a) Optical spectrum and (b) autocorrelation trace of the PMLL with sidebands injection-locking; injection power $\mathrm{P}_{\text {inj }}=240 \mu \mathrm{W}$. (c) Optical spectrum and (d) autocorrelation trace of the PMLL with sidebands injection-locking; injection power $\mathrm{P}_{\text {inj }}=570 \mu \mathrm{W}$.

linewidth reduction for the slave. The injection power, $\mathrm{P}_{\mathrm{inj}}$, had a major influence on the slave characteristics. Two locking regimes were found, with wider and narrower spectral widths. Examples of optical spectra and corresponding autocorrelation traces for the two observed regimes are shown in Figure 5.

As expected, the regime with fewer modes allowed $F_{\text {rep }}$ tuning in a larger range and resulted in a lower jitter. ${ }^{12}$ For comparison, single sideband phase noise measurements in the range from $10 \mathrm{kHz}$ to $1 \mathrm{GHz}$ for the HMLL (black solid line), HMLL with injection seeding (red dashed line), and PMLL with dual-tone injection (blue dotted line) at their respective optimal operating point are shown in Figure 6(a). For dual-tone injection seeding, a strongly suppressed phase noise is observed over the entire frequency range of the carrier offset, resulting in a decisively reduced jitter of $121 \mathrm{fs}$. Figure $6(\mathrm{~b})$ shows $F_{\text {rep }}$ tuning range (red triangles) and timing jitter (black circles) of the sidebands injection-locked PMLL as a function of sideband injection power. For $570 \mu \mathrm{W}$, an integrated jitter of 121 fs along with a record $F_{\text {rep }}$ tuning range of $342 \mathrm{MHz}$ has been achieved. However, the spectral width reduction led to the pulse width broadening to a value of 5.2 ps (Figure 5(d)).

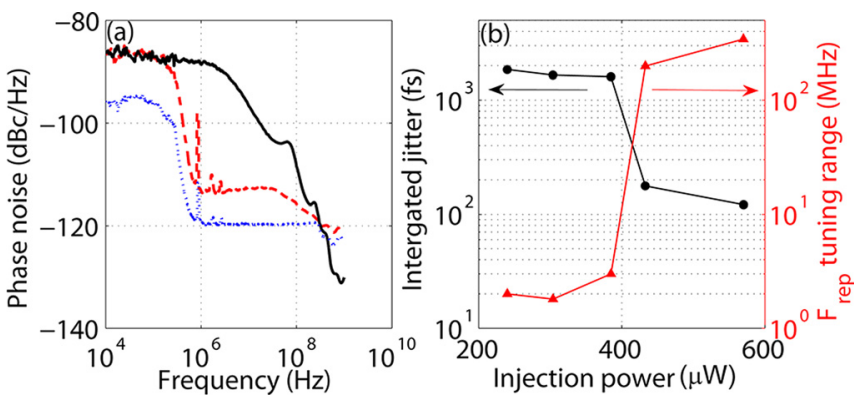

FIG. 6. (a) Single sideband phase noise in the range from $10 \mathrm{kHz}$ to $1 \mathrm{GHz}$ for the HMLL (black solid line), HMLL with injection seeding (red dashed line), and PMLL with dual-tone injection (blue dotted line). (b) $F_{\text {rep }}$ tuning range (red triangles) and integrated jitter (black circles) of the PMLL with sidebands injection-locking. 
Several techniques allowing repetition rate tuning and noise reduction in MLLs has been studied on the same device. Hybrid mode-locking resulted in jitter reduction to 620 fs and $F_{\text {rep }}$ tuning range of $10 \mathrm{MHz}$ for the chosen bias conditions. Hybrid mode-locking had no significant impact on the device optical spectrum and modal linewidth; autocorrelation traces were slightly broader (3.1 ps) when compared to the PMLL (2.8 ps).

Hybrid modulation with $\mathrm{CW}$ injection and dual-tone injection seeding of the PMLL had similar outcomes, resulting in linewidth narrowing for all laser modes, RF linewidth reduction below the resolution bandwidth of the ESA, and the optical spectrum narrowing and red shifting. An increase of the injection power resulted in a wider $F_{\text {rep }}$ tuning range and lower jitter values due to reduction in the number of lasing modes. Hybrid modulation with injection resulted in the jitter of $240 \mathrm{fs}$ and $167 \mathrm{MHz}$ tuning range. The lowest integrated jitter of $121 \mathrm{fs}$ and the widest $F_{\text {rep }}$ tuning range of $342 \mathrm{MHz}$ were achieved with dual-tone injection seeding.

This work was funded by the EU FP7 Marie Curie Action FP7-PEOPLE-2010-ITN through the PROPHET project, Grant No. 264687, by the INSPIRE programme, funded by the Irish Government's Programme for Research in Third Level Institutions, Cycle 4, National Development Plan 2007-2013, by Science Foundation Ireland under Contract No. 07/IN.1/I929 and from the DFG in the framework of the SFB 787. T.H. acknowledges support of the EU FP7 Marie Curie Action FP7-PEOPLE-2011-IEF, Grant No. 299288.

${ }^{1}$ A. Gubenko, D. A. Livshits, I. L. Krestnikov, S. S. Mikhrin, A. A. Kozhukhov, A. R. Kovsh, N. N. Ledentsov, A. E. Zhukov, and E. L. Portnoi, Electron. Lett. 41(20), 1124 (2005).

${ }^{2}$ M. Laemmlin, G. Fiol, C. Meuer, M. Kuntz, F. Hopfer, A. R. Kovsh, N. N. Ledentsov, and D. Bimberg, Electron. Lett. 42, 697 (2006).

${ }^{3}$ E. U. Rafailov, M. A. Cataluna, and W. Sibbett, Nat. Photonics 1, 395 (2007).

${ }^{4}$ M. A. Cataluna, E. U. Rafailov, A. D. McRobbie, W. Sibbett, D. A. Livshits, and A. R. Kovsh, IEEE Photonics Technol. Lett. 18, 1500 (2006).

${ }^{5}$ M. Sugawara and M. Usami, Nat. Photonics 3, 30 (2009).
${ }^{6}$ M. G. Thompson, K. T. Tan, C. Marinelli, K. A. Williams, R. V. Penty, I. H. White, M. Kuntz, D. Ouyang, D. Bimberg, V. M. Ustinov, A. E. Zhukov, A. R. Kovsh, N. N. Ledentsov, D.-J. Kang, and M. G. Blamire, Electron. Lett. 40, 346 (2004).

${ }^{7}$ M. G. Thompson, A. R. Rae, M. Xia, R. V. Penty, and I. H. White, IEEE J. Sel. Top. Quantum Electron. 15, 661 (2009).

${ }^{8}$ M. Costa e Silva, A. Lagrost, L. Bramerie, M. Gay, P. Besnard, M. Joindot, J. C. Simon, A. Shen, and G. H. Duan, in Proceedings of Optical Fiber Communication $(O F C)$, collocated National Fiber Optic Engineers Conference, 2010 Conference on (OFC/NFOEC), Lannion, France, 21-25 March 2010, pp. 1-3.

${ }^{9}$ C. Liu, H. Cao, G. A. Smolyakov, P. G. Eliseev, and M. Osinski, Electron. Lett. 41, 963 (2005).

${ }^{10}$ M. G. Thompson, C. Marinelli, K. T. Tan, K. A. Williams, R. V. Penty, I. H. White, I. N. Kaiander, R. L. Sellin, D. Bimberg, D. J. Kang, M. G. Blamire, F. Visinka, S. Jochum, and S. Hansmann, Electron. Lett. 39, 1121 (2003).

${ }^{11}$ M. G. Thompson, D. Larsson, A. Rae, K. Yvind, R. V. Penty, I. H. White, J. Hvam, A. R. Kovsh, S. S. Mikhrin, and I. L. Krestnikov, in Proceedings of Eur. Conference on Optics Communication (ECOC), Cannes, France, 24-28 September, 2006, pp 1-2.

${ }^{12}$ G. Fiol, D. Arsenijevic, D. Bimberg, A. G. Vladimirov, M. Wolfrum, E. A. Viktorov, and P. Mandel, Appl. Phys. Lett. 96, 011104 (2010).

${ }^{13}$ A. Takada and W. Imajuku, IEEE Photonics Technol. Lett. 9(10), 1328 (1997).

${ }^{14}$ M. Teshima, K. Sato, and M. Koga, J. Quantum Electron. 34(9), 1588 (1998).

${ }^{15}$ T. Jung, J.-L. Shen, D. T. K. Tong, S. Murthy, M. C. Wu, T. Tanbun-Ek, W. Wang, R. Lodenkamper, R. Davis, L. J. Lembo, and J. C. Brock, IEEE Trans. Microwave Theory Tech. 47(7), 1225 (1999).

${ }^{16}$ J. Kim and P. J. Delfyett, Opt. Express 16, 11153 (2008).

${ }^{17}$ D. Goulding, S. P. Hegarty, O. Rasskazov, S. Melnik, M. Harnett, G. Greene, J. G. McInerney, D. Rachinskii, and G. Huyet, Phys. Rev. Lett. 98, 153903 (2007).

${ }^{18}$ T. Habruseva, S. P. Hegarty, A. Vladimirov, A. Pimenov, D. Rachinskii, N. Rebrova, E. Viktorov, and G. Huyet, Opt. Express 20(23), 25572 (2012).

${ }^{19}$ T. Habruseva, G. Huyet, and S. P. Hegarty, IEEE J. Sel. Top. Quantum Electron. 17(5), 1272 (2011).

${ }^{20}$ L. Goldberg, H. F. Taylor, and J. F. Weller, Electron. Lett. 18(11), 1019 (1982).

${ }^{21}$ T. Habruseva, S. O’Donoghue, N. Rebrova, D. A. Reid, L. Barry, D. Rachinskii, G. Huyet, and S. P. Hegarty, IEEE Photon. Technol. Lett. 22(6), 359 (2010).

${ }^{22}$ T. Habruseva, N. Rebrova, S. P. Hegarty, and G. Huyet, Quantum Dot Devices, Lecture Notes in Nanoscale Science and Technology (SpringerVerlag, New York, 2012), Vol. 13, p. 65-91. 